\title{
SELF-COMPLEMENTARY GRAPH DECOMPOSITIONS
}

\author{
C. A. RODGER
}

(Received 21 March 1990)

Communicated by L. Caccetta

\begin{abstract}
A complementary decomposition of $\lambda K_{n}$ into a graph $G$ is an edge-disjoint decomposition of $\lambda K_{n}$ into copies of $G$ such that if each copy $H$ of $G$ is replaced by its complement in $V(H)$ then the result is an edge-disjoint decomposition of $\lambda K_{n}$ into copies of $G^{c}$; it is a selfcomplementary decomposition if $G=G^{c}$. The spectrum for the last self-complementary graph on at most 7 vertices is found.
\end{abstract}

1991 Mathematics subject classification (Amer. Math. Soc.): 05 B 30, 05 B 40, 05 C 70.

\section{Introduction}

A $G$-design (of $\lambda K_{n}$ ) is an ordered triple $(V, B, \lambda)$ where $V$ is the vertex set of $\lambda K_{n} \quad(n=|V|)$ and $B$ is a collection of graphs, each isomorphic to $G$, which form an edge-disjoint decomposition of $\lambda K_{n} ; n$ is called the order and $\lambda$ is called the index of the $G$-design. Let $C_{m}$ denote a cycle of length $m$. Let $H^{c}$ be the complement of $H$ in $V(H)$.

In recent years, much attention has been focussed on $G$-designs and on $G$ designs with additional properties. For example, $K_{m}$-designs are just block designs, and $C_{m}$-designs have also been called balanced cycle designs and $m$-cycle systems. Perhaps the most natural question to ask about $G$-design is what is their spectrum, that is, for which values of $n$ do they exist? In the case where $G=C_{m}$, the spectrum remains unknown, despite having been considered for at least 25 years (see [4] for example). More recently the

This research was supported by NSF grant DMS-8805475.

(C) 1992 Australian Mathematical Society 0263-6115/92 \$A2.00+0.00 
existence problem has been settled in the cases where $G$ is a path [11], and where $G$ is a star [10], and has nearly been settled when $G$ is a graph with at most 5 vertices [1].

Related to this problem is the existence problem for $G$-designs that satisfy additional properties. For example, the spectra for $C_{m}$-designs that are resolvable, almost resolvable, or $i$-perfect have been successfully studied. Similar results exist when $G$ is a star or a path. For a survey, see [7].

In this paper we consider the spectrum problem for self-complementary $G$-designs. A complementary $G$-design is a $G$-design $(V, B, \lambda)$ with the additional property that replacing each copy $H$ of $G$ in $B$ by its complement in $V(H)$ results in a $G^{c}$-design. For example if $\lambda=1$ and $G=K_{1,3}$ then $G^{c}=K_{3}$ (together with an isolated vertex), so complementary $K_{1,3^{-}}$ designs are equivalent to nested Steiner triple systems; the spectrum for these has been found [8]. A self-complementary $G$-design is a complementary $G$ design in which $G \cong G^{c}$. For example, if $G=P_{3}$, a path of length 3 , then $G=G^{c}$; the spectrum problem for self-complementary $P_{3}$-designs has been found when $\lambda=1[2,6]$. The spectrum for self-complementary $C_{5}$-designs (also known as Steiner pentagon systems) has also been found [5]. Here we consider the remaining self-complementary graph with at most 7 vertices.

Let $M$ be the graph with $V(M)=\{a, b, c, d, e\}$ and $E(M)=\{a b, b c$, $b d, c d, d e\}$; throughout this paper we shall denote $M$ by $(a, b, c, d, e)$. Then $M \cong M^{c}$. The purpose of this paper is to find the spectrum for selfcomplementary $M$-designs, for all $\lambda$.

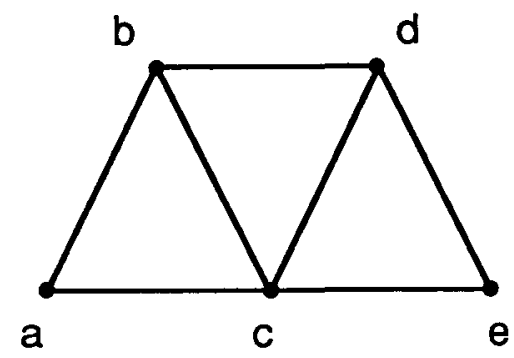

The graph $M=(a, b, c, d, e)$.

\section{Preliminary results}

We shall make use of quasigroups with various properties in constructing the self-complementary $M$-designs. The properties that are not defined here are well known, but can be found in [9]. Let $Z_{x}=\{0,1, \ldots, x-1\}$, and let $(a, b, c, d, e)+i=(a+i, b+i, c+i, d+i, e+i)$. 
LeMma 2.1. For $n>4, n \notin\{6,10\}$, there exist 3 idempotent mutually orthogonal quasigroups of order $n$.

LEMMA 2.2 [13]. For all odd $n \geq 5$ there exists an idempotent self-orthogonal quasigroup of order $n$ which is orthogonal to an idempotent commutative quasigroup.

Let $h_{i}=\{2 i, 2 i+1\}$ and let $H=\left\{h_{i} \mid 0 \leq i \leq s-1\right\}$; the elements of $H$ are called holes. A self-orthogonal quasigroup with holes $H$ is a quasigroup $\left(Z_{2 s}, \cdot\right)$ in which

(a) for $0 \leq i \leq s-1,2 i \cdot 2 i=2 i=(2 i+1) \cdot(2 i+1)$ and $(2 i+1) \cdot 2 i=$ $2 i+1=2 i \cdot(2 i+1)$, and

(b) for all $(x, y) \in\left(Z_{2 s} \times Z_{2 s}\right) \backslash\left(\bigcup_{i=0}^{s-1}\left(h_{i} \times h_{i}\right)\right)$ there exists a unique pair $i$ and $j$ such that $i \cdot j=x$ and $j \cdot i=y$.

A self-orthogonal quasigroup with holes $H,\left(Z_{2 s}, \cdot\right)$ is orthogonal to a commutative quasigroup with holes $H,\left(Z_{2 s}, \circ\right)$ if for all $(x, y) \in\left(Z_{2 s} \times\right.$ $\left.Z_{2 s}\right) \backslash\left(\bigcup_{i=0}^{s-1}\left(h_{i} \times h_{i}\right)\right)$ there exists a unique pair $i$ and $j$ such that $i \cdot j=x$ and $i \circ j=y$.

LEMMA 2.3 [12]. For all $n \equiv 2(\bmod 4), n \notin\{6,30,66,174\}$ there exists a self-orthogonal quasigroup with holes $H$ that is orthogonal to a commutative quasigroup with holes $H$.

Clearly self-complementary $M$-designs bear some relation to $K_{5}$-designs (block designs with block size 5). We shall use the following result of Hanani.

LEMMA 2.4 [3]. For all $n \equiv 1$ or $5(\bmod 20)$ there exists a $K_{5}$-design of $K_{n}$. There does not exist a $K_{5}$-design of $2 K_{15}$.

We will need some small $M$-designs.

Lemma 2.5. There exist self-complementary $M$-designs of $K_{n}$ for $n \in$ $\{5,11,31\}$.

Proof. $\left(Z_{5},\{(0,1,2,3,4),(1,4,2,0,3)\}, 1\right)$ is a self-complementary $M$-design of $K_{5} \cdot\left(Z_{11},\{(0,4,1,2,7)+i \mid 0 \leq i \leq 10\}, 1\right)$ is a selfcomplementary $M$-design of $K_{11}$ (reducing all sums modulo 11 ).

$\left(Z_{31},\{(0,1,5,17,8)+i,(0,2,20,12,18)+i,(0,5,22,25,1)+i \mid 0\right.$ $\leq i \leq 30\}, 1$ ) is a self-complementary $M$-design of $K_{31}$ (reducing all sums modulo 31). 
LEMMA 2.6. There exist self-complementary $M$-designs of $2 K_{n}$ for $n \in$ $\{6,10,15,16,20,30\}$.

Proof.

$$
\begin{aligned}
& n=6: \quad\left(Z_{6},\{(0,4,1,2,3)+i \mid 0 \leq i \leq 5\}, 2\right) \\
& n=10: \quad\left(\{\infty\} \cup Z_{9},\{(0,3,5,6,1)+i,(1,2, \infty, 0,4)+i \mid 0 \leq i \leq 8\}, 2\right) \\
& n=15: \quad\left(\{\infty\} \cup Z_{14},\{(0,1,2,4,6)+i \text {, }\right. \\
& (0,3,7,12,6)+i,(0,4, \infty, 11,5)+i \mid 0 \leq i \leq 13\}, 2) \\
& n=16: \quad\left(Z_{16},\{(0,1,2,4,7)+i,(0,2,7,14,6)+i,(0,5,11,1,8)\right. \\
& +i \mid 0 \leq i \leq 15\}, 2) \\
& n=20: \quad\left(\{\infty\} \cup Z_{19},\{(0,1,2,4,6)+i,(0,3,7,11,16)+i,\right. \\
& (0,5,11,18,10)+i,(0,9, \infty, 2,12)+i \mid 0 \leq i \leq 18\}, 2) \\
& n=30:\left(\{\infty\} \cup Z_{29},\{(0,1,5,11,20)+i,(0,3,14,7,20)+i \text {, }\right. \\
& (0,1,3,6,4)+i,(0,5,11,21,13)+i,(0,7,19,27,12) \\
& +i,(0,12, \infty, 1,16)+i \mid 0 \leq i \leq 28\}, 2)
\end{aligned}
$$

Finally, we note the following necessary conditions.

LeMma 2.7. If there exists a self-complementary $M$-design of $\lambda K_{n}$ then

(a) if $\lambda \equiv 1,3,7$ or $9(\bmod 10)$ then $n \equiv 1$ or $5(\bmod 10)$, and if $\lambda=1$ then $n \neq 15$,

(b) if $\lambda \equiv 2,4,6$ or $8(\bmod 10)$ then $n \equiv 0$ or $1(\bmod 5)$,

(c) if $\lambda \equiv 5(\bmod 10)$ then $n \equiv 1(\bmod 2), n \neq 3$,

(d) if $\lambda \equiv 0(\bmod 10)$ then $n \notin\{2,3,4\}$.

Proof. If there exists a complementary $M$-design of $\lambda K_{n}$ then there exists a $K_{5}$-design of $2 \lambda K_{n}$. Therefore, by Lemma 2.4 , if $\lambda=1$ then $n \neq 15$. The rest of the lemma follows from straightforward counting arguments.

\section{The case $\lambda=1$}

THEOREM 3.1. Let $n \equiv 5(\bmod 10)$. There exists a self-complementary $M$-design of $K_{n}$ except if $n=15$.

Proof. Let $n=10 s+5=5(2 s+1)$. By Lemmas 2.5 and 2.7 we can assume that $2 s+1 \geq 5$. Let $\left(Z_{2 s+1}, \cdot\right)$ be an idempotent self-orthogonal quasigroup that is orthogonal to the idempotent commutative quasigroup 
$\left(Z_{2 s+1}, 0\right)$ (these quasigroups exist by Lemma 2.2). Then define a selfcomplementary $M$-design $\left(Z_{5} \times Z_{2 s+1}, B, 1\right)$ as follows:

(a) for $0 \leq i \leq 2 s$, let $\{((0, i),(1, i),(2, i),(3, i),(4, i)),((1, i)$, $(4, i),(2, i),(0, i),(3, i))\} \subseteq B$, and

(b) for $0 \leq i<j \leq 2 s$, and for $0 \leq r \leq 4$, let $((2+r, i \cdot j),(r, i),(1+$ $r, i \circ j),(r, j),(2+r, j \cdot i)) \in B$, (where the sums in the first coordinate are reduced modulo 5 ).

The fact that this defines an $M$-design easily follows from the fact that $\left(Z_{2 s+1}, 0\right)$ is a quasigroup and that $\left(Z_{2 s+1}, \cdot\right)$ is an idempotent commutative quasigroup. The orthogonality of the quasigroups ensures that together the complements of each copy of $M$ form an $M$-design.

To see this, notice that the complements of the copies of $M$ in (a) produce the same set of copies of $M$. The complement of the graphs defined in (b) are

$$
\{(r, j),(2+r, i \cdot j),(1+r, i \circ j),(2+r, j \cdot i),(r, i)\}
$$

for $0 \leq i<j \leq 2 s$ and $0 \leq r \leq 4$. So, for example, the edge $\{(a, b),(a, c)\}$ is in the graph $(*)$ where $i \cdot j=b$ and $j \cdot i=c$; there is exactly one such choice for $i<j$ by the self-orthogonality of $\left(Z_{2 s+1}, \cdot\right)$. Similarly the edge $\{(a, b),(a+1, c)\}$ is in the graph (*) with $i \circ j=b$ and $i \cdot j=c$ (or $j \cdot i=c)$; there is exactly one such choice for $i<j$ by the orthogonality of $\left(Z_{2 s+1}, o\right)$ and $\left(Z_{2 s+1}, \cdot\right)$. The remaining details are left to the reader.

THEOREM 3.2. Let $n \equiv 11(\bmod 20)$. There exists a self-complementary $M$-decomposition of $K_{n}$ except possibly if $n \in\{151,331,871\}$.

Proof. Let $n=20 s+11=5(4 s+2)+1$. Using Lemma 2.5 we can assume that $s \geq 2$. Let $\left(Z_{4 s+2}, \cdot\right)$ be a self-orthogonal quasigroup with holes $\{\{2 x, 2 x+1\} \mid 0 \leq x \leq 2 s\}$ that is orthogonal to $\left(Z_{4 s+2}, 0\right)$, a commutative quasigroup with holes $\{\{2 x, 2 x+1\} \mid 0 \leq x \leq 2 s\}$ (these quasigroups exist by Lemma 2.3 ). Define a self-complementary $M$-design $\left(\{\infty\} \cup\left(Z_{5} \times Z_{4 s+2}\right), B, 1\right)$ as follows:

(a) for $0 \leq x \leq 2 s$, place a copy of the self-complementary $M$-design in Lemma 2.5 on the vertices $\{\infty\} \cup\left(Z_{5} \times\{2 x, 2 x+1\}\right)$ in $B$, and

(b) for $0 \leq i<j \leq 4 s+1,\{i, j\} \notin\{\{2 x, 2 x+1\} \mid 0 \leq x \leq 2 s\}$ and for $0 \leq r \leq 4$ let $((2+r, i \cdot j),(r, i),(1+r, i \circ j),(r, j),(2+r, j \cdot i)) \in B$.

The fact that this defines a self-complementary $M$-design follows in the same way as the proof of Theorem 3.1.

Lemma 3.3. Let $n \equiv 1(\bmod 20)$. There exists a self-complementary $M$ design of $K_{n}$. 
Proof. By Lemma 2.4 there exists a $K_{5}$-design of $K_{n}$; replace each copy of $K_{5}$ with the self-complementary $M$-design of $K_{5}$ in Lemma 2.5.

Lemma 3.4. If there exist self-complementary $M$-designs of $K_{m}$ and of $K_{n+1}$ and if there exist 3 orthogonal quasigroups of order $n$ then there exists a self-complementary $M$-design of $K_{m n+1}$.

Proof. Let $\left(Z_{n}, \cdot_{1}\right),\left(Z_{n}, \cdot_{2}\right)$ and $\left(Z_{n}, \cdot_{3}\right)$ be 3 orthogonal quasigroups. Let $\left(Z_{m}, B_{1}, 1\right)$ be a self-complementary $M$-design of $K_{m}$ and for each $i \in Z_{m}$ let $\left(\{\infty\} \cup\left(\{i\} \times Z_{n}\right), B(i), 1\right)$ be a self-complementary $M$-design of $K_{n+1}$. Then $\left(\{\infty\} \cup\left(Z_{m} \times Z_{n}\right), B, 1\right)$ is a self-complementary $M$-design of $K_{m n+1}$, where

$$
\begin{array}{r}
B=\bigcup_{i \in Z_{m}} B_{i} \cup\left\{\left((a, i),(b, j),\left(c, i \cdot{ }_{1} j\right),\left(d, i \cdot{ }_{2} j\right),\left(e, i \cdot{ }_{3} j\right)\right) \mid i \in Z_{n},\right. \\
\left.j \in Z_{n},(a, b, c, d, e) \in B_{1}\right\} .
\end{array}
$$

COROLlaRY 3.5. There exist self-complementary $M$-designs of $K_{151}, K_{331}$ and $K_{871}$.

Proof. Apply Lemma 3.4 with $(m, n)=(5,30),(11,30)$ and $(5,174)$ respectively.

THEOREM 3.6. The spectrum for self-complementary $M$-designs of index 1 is $n \equiv 1$ or $5(\bmod 10), n \neq 15$.

Proof. This follows from Lemmas 2.7, 3.3 and 3.4, Corollary 3.5 and Theorems 3.1 and 3.2.

\section{The cases $\lambda>1$}

TheOREM 4.1. Let $n \equiv 0$ or $1(\bmod 5)$. There exists a self-complementary $M$-design of $2 K_{n}$.

Proof. Of course if there exists a self-complementary $M$-design of $K_{n}$ then there also exists one of $2 K_{n}$. For $n \in\{6,10,15,16,20,30\}$ selfcomplementary $M$-designs of $2 K_{n}$ are constructed in Lemma 2.6. For $n=$ 50 , modify the construction in Theorem 3.2 with $s=2$ by using a copy of the self-complementary $M$-design of $2 K_{10}$ in (a) and taking two copies of each of the blocks in (b); this produces a self-complementary $M$-design of $2 K_{50}$ on the vertex set $Z_{5} \times Z_{10}$. 
In any other case, $n=5 s$ or $5 s+1$ where $s$ is an integer for which there exist 3 idempotent mutually orthogonal quasigroups of order $s$, say $\left(Z_{s}, \cdot_{1}\right)$, $\left(Z_{s}, \cdot_{2}\right)$ and $\left(Z_{s}, \cdot_{3}\right)$ (see Lemma 2.1). If $n=5 s$ then a self-complementary $M$-design $\left(Z_{5} \times Z_{s}, B, 2\right)$ can be formed as follows:

(a) for $0 \leq x \leq s-1, B$ contains a copy of a self-complementary $M$ design of $2 K_{5}$ on the vertex set $Z_{5} \times\{x\}$, and

(b) for $0 \leq i \leq s-1,0 \leq j \leq s-1, i \neq j$ and $0 \leq r \leq 4$ let $\left(\left(2+r, i \cdot{ }_{1} j\right),(r, i),\left(1+r, i \cdot{ }_{2} j\right),(r, j),\left(2+r, i \cdot{ }_{3} j\right)\right) \in B$.

If $n=5 s+1$ then a self-complementary $M$-design $\left(\{\infty\} \cup\left(Z_{5} \times Z_{s}\right), B, 2\right)$ can be produced by using a self-complementary $M$ design of $2 K_{6}$ on the vertex set $\{\infty\} \cup\left(Z_{5} \times\{x\}\right)$ in part (a) above.

The fact that these constructions produce self-complementary $M$-designs follows in the same way as the proof of Theorem 3.1.

THEOREM 4.2. Let $n \equiv 1(\bmod 2)$. For all $n \neq 3$ there exists a selfcomplementary $M$-design of $5 K_{n}$.

Proof. Let $n=2 s+1$. By Lemma 2.2 there exists an idempotent selforthogonal quasigroup $\left(Z_{2 s+1}, \cdot\right)$ that is orthogonal to an idempotent commutative quasigroup $\left(Z_{2 s+1}, 0\right)$. Then $\left(Z_{2 s+1},\{(i \cdot j, i, i \circ j, j, j \cdot i) \mid 0 \leq\right.$ $i<j \leq 2 s\}, 5)$ is a self-complementary $M$-design of $5 K_{n}$.

THEOREM 4.3. For all $n \geq 5$ there exists a self-complementary $M$-design of $10 K_{n}$.

Proof. For $n=6$ or 10 , such a design can be produced by taking 5 copies of the designs in Lemma 2.6. For any other $n$, by Lemma 2.1 there exist 3 idempotent mutually orthogonal quasigroups of order $n$, say $\left(Z_{n}, \cdot_{1}\right)$, $\left(Z_{n}, \cdot_{2}\right)$ and $\left(Z_{n}, \cdot_{3}\right)$. Then $\left(Z_{n},\left\{\left(i \cdot \cdot_{1} j, i, i \cdot \cdot_{2} j, j, i \cdot{ }_{3} j\right) \mid\{i, j\} \subseteq Z_{n}\right.\right.$, $i \neq j\}, 10)$ is a self-complementary $M$-design of $10 K_{n}$.

\section{Conclusions}

The results in the previous sections can be combined to give the following theorem.

THEOREM 5.1. The necessary conditions in Lemma 2.7 for the existence of a self-complementary $M$-design of $\lambda K_{n}$ are sufficient.

Proof. This follows immediately from Lemma 2.7 and Theorems 3.6, 4.1, 4.2 and 4.3 by combining self-complementary $M$-designs with $\lambda \epsilon$ 
$\{1,2,5,10\}$ to obtain self-complementary $M$-designs for other values of $\lambda$, except when $\lambda=3$ and $n=15$. Recently a self-complementary $M$-design of $3 K_{15}$ was constructed by Elizabeth J. Billington (private communication).

\section{References}

[1] J. C. Bermond, C. Huang, A. Rosa and D. Sotteau, 'Decompositions of complete graphs into isomorphic subgraphs with five vertices,' Ars Combinatoria 10 (1980), 211-254.

[2] A. Granville, A. Moisiadis and R. Rees, 'On Complementary Decompositions of the complete graph,' Graphs and Combinatorics 5 (1989), 57-62.

[3] H. Hanani, 'Balanced incomplete block designs and related designs,' Discrete Math. 11 (1975), 255-369.

[4] D. G. Hoffman, C. C. Lindner and C. A. Rodger, 'On the construction of odd cycle systems,' J. Graph Th. 13 (1989), 417-426.

[5] C. C. Lindner and D. R. Stinson, 'Steiner pentagon systems,' Discrete Math. 52 (1984), 67-74.

[6] R. Rees and C. A. Rodger, 'Subdesigns in complementary path decompositions and incomplete two-fold designs with block size four,' Ars Combinatoria, to appear.

[7] C. A. Rodger, 'Graph decompositions,' Le Matematiche 45, (1990), 119-139.

[8] D. R. Stinson, 'The spectrum of nested Steiner triple systems,' Graphs and Combinatorics 1 (1985), 189-191.

[9] A. P. and D. J. Street, Combinatorics of experimental design, Oxford Univ. Press, 1987.

[10] M. Tarsi, 'Decompositions of complete multigraphs into stars,' Discrete Math. 26 (1979), 273-278.

[11] M. Tarsi, 'Decompositions of a complete multigraph into simple paths: non-balanced handcuff designs', J. Combinatorial Th. (A) 34 (1983),60-70.

[12] L. Zhu, 'Existence of Holey Solssoms of Type $2^{n}$,' Congressus Numerantium 45 (1984), 295-304.

[13] L. Zhu, 'A few more self-orthogonal latin squares with symmetric orthogonal mates,' Proc. 13th Conf. on Num. Math. and Computing, University of Manitoba, 1983.

120 Mathematics Annex

Auburn University

Auburn, AL 36849-5307

USA 